Journal of Computer Science 7 (12): 1760-1764, 2011

ISSN 1549-3636

(C) 2011 Science Publications

\title{
Gradient Descent Learning for Utility Current Compensation using Active Regenerative PWM Filter
}

\author{
${ }^{1}$ R. Balamurugan and ${ }^{2} \mathrm{G}$. Gurusamy \\ ${ }^{1}$ KSR College of Technology, Tiruchengode-637215, Tamil Nadu, India \\ ${ }^{2}$ Bannari Amman Institute of Technology, \\ Sathyamangalam-638401, Tamil Nadu, India
}

\begin{abstract}
Problem statement: Harmonic analysis is a primary matter of power quality assessment. Its main intention is to check the utility whether it is delivering the loads without any deviations in voltages and currents. The problem is due to proliferation of Electronic converters and power electronics which gave birth to numerous new applications, offering unmatched comfort to the customers. Approach: Harmonics should be maintained within the limits said in standards like IEEE 519 and others such as IEEE 1159 for safeguarding the utility. This was provided by many mitigation technologies like passive, shunt and series filtering, active conditioners, but they were lack of some demerits like huge cost, many controllers and circuit components. So for controlling the harmonic loads the converter with four quadrant characteristics was implemented and this converter act as shunt active filter as well as rectifier simultaneously without any additional circuitry. For having better harmonic reduction in addition, many controllers like p-q Theorem based controller, Fuzzy and gradient descent based neural network is also used. Results: The simulation results gives the compared source current wave forms for various controllers with individual harmonic mitigations. Conclusion: The pure utility current is obtained by using this intelligent neural filter without any additional components and without any extra controllers than the conventional methods.
\end{abstract}

Key words: Harmonics, nonlinear loads, solid-state electronics, Total Harmonic Distortion (THD), Active Regenerative Filters (ARF), p-q theorem based controller, Fuzzy Logic Controller (FLC), gradient descent back propagated neural network

\section{INTRODUCTION}

Solid-state electronics is based on the use of semiconductors. These materials are totally different in their response to voltage and current. This is lead to misleading of both because each solid-state device will have a unique response curve that is different from that of other types of semiconductor-based devices (Ghosh and Narayanan, 2005). So for a nonlinear load, the relationship between voltage and current cannot be predicted easily with equipment containing many solidstate devices. The only logical way is to use test instruments to plot the individual voltage-current relationships. The test results are often baffling. With an incoming source having a near perfect $50 \mathrm{~Hz}$ sine wave, the current will be significantly distorted. However, mathematical (Fourier) analysis of these distorted waves shows that they are made up of the fundamental sine wave plus one or more harmonic current waves having a frequency that is a whole integer multiple of the fundamental frequency (Rabi and Arumugam, 2005). For example, a $50 \mathrm{~Hz}$ fundamental, combined with 150 and $250 \mathrm{~Hz}$ waves, will result in a specific type of distorted wave (IEEE, 1993). Generally two types of loads, they are linear and nonlinear. When the load current is proportional to the applied voltage, then the loads are linear. Motors, incandescent lighting and heating loads are linear in nature. Nonlinear loads, on the other hand, are loads in which the load current is not proportional to the instantaneous voltage and here the load current is often not continuous. This is the result of the nonlinear load being switched on for only part of the cycle, as in a thyristor-controlled circuit, or pulsed, as in a controlled-rectifier circuit (García et al., 2003).

Corresponding Author: R. Balamurugan, KSR College of Technology, Tiruchengode-637215, Tamil Nadu, India 
The nonlinear loads, such as inverters, solid-state rectifiers used in welders, DC power supplies, variablefrequency drives and electronic ballasts for lighting are sources of harmonics in the electrical system (Rodriguez et al., 2005). The uneven switching of converters also acts as nonlinear loads for generation of harmonics.

The magnitude of the current and voltage waveform distortion will depend upon the relative size of the nonlinear loads with respect to that system, the type of equipment and the source impedance (Salmon, 1993). The amount of voltage distortion increases as the percentage of nonlinear loads increases (Benachaiba and Ferdi, 2009). These generated harmonics should be reduced within the reasonable limits as said in standards like IEEE 519 (IEEE, 1993) to safeguard the utilities and other systems connected to it.

Harmonic observations: The testing parameters are input voltage, input current, $\mathrm{THD}_{\mathrm{i}}$, power factor and power. The 1KVA and 5KVA traditional UPS are tested with linear load (resistive lamp loads) and nonlinear loads like personal computer with SMPS. The THDi is measured and waveforms are taken by using fluke meters with linear and nonlinear load and shown in Fig. 1a-d.

The results reveal that they contain more harmonics for nonlinear loads compared with linear loads like resistive lamp loads. These harmonics rate should be reduced to the limit as said in the IEEE standards (less than 5\%).

Software simulation with mitigation of harmonics: The simulink modeling of the nonlinear load is done by using MATLAB software. The modeling extends to the uncompensation of harmonic sources and testing of controllers with respect to their controllability. By the results obtained, the effectiveness of the controllers is validated. The nonlinear load like uneven switching of single phase half bridge converter is taken for software simulation and verification.

After simulation, the harmonics obtained from the nonlinear load without compensation is $190.55 \%$ $\left(\mathrm{THD}_{\mathrm{i}}\right)$. This THD is reduced by using the controllers like $\mathrm{p}-\mathrm{q}$ theorem based current controller, fuzzy logic tuned controller and neuro trained controller. The uncompensated profile of harmonics with the FFT (Fast Fourier Transform) window wave forms of two selected cycles of source current are shown below in Fig. 2a-b for the above nonlinear load.

\section{MATERIALS AND MEHTODS}

p-q theorem based controller with ARF: The model of control is Active Regenerative filter i.e., VSCC PWM (Voltage Source Current Controlled Pulse Width Modulated) rectifier acting simultaneously as shunt active filter without any additional circuitry. This is possible by using the above regenerative rectifier whenever the nonlinear loads are connected between this converter and current sensors (Kazerani et al., 1991). The above said current controlled converter detects the typical nonlinear load and makes the supply current to sinusoidal. Thus the regenerative rectifier shows the four quadrant characteristics. In order to wave shape the current different controllers are used to control this Active Regenerative Rectifier (ARF).

For the first stage of testing the effectiveness of the controlling, the $\mathrm{p}-\mathrm{q}$ theorem based current controller is used. Here in controlling of harmonics, the extension p$\mathrm{q}$ theorem is used for deriving the sinusoidal current reference template (Chen and Pan, 1992). This reference current in addition with a High Pass Filter (HPF) gives the reduction of higher order harmonics especially (Tan et al., 2005).

Fuzzy tuned controller with ARF: The FL control is simulated for observations better than the $\mathrm{p}-\mathrm{q}$ theorem based controller. The basic FLC operation for obtaining the required results determines the fuzzy rules applied to it. The fuzzy characteristics are with Mamdani Fuzzy Inference System is used. The defuzzification is centroid. This inference system gives the rules framed for controlling the harmonics with respect to the data base available. The Active regenerative rectifier gets the controlled switching by using the above required control mechanism.

The reference sine template is generated and it is controlled with the dc link voltage of regenerative converter (Chandr et al., 2000). By doing so the current with opposite harmonics $\left(I_{c}\right)$ is injected by rectifier into the line. This injected anti harmonic current $I_{c}$ when added with $\mathrm{I}_{\mathrm{L}}$ gives pure sine wave source at the utility thereby elimination of the harmonics (Valdez et al., 2009). The fuzzy characteristics are (Sivanandan and Deepa, 2008) :

- Triangular membership functions

- Fuzzification using continuous universe of discourse

- Implication using the "min" operator

- Inference mechanism based on fuzzy implication

- Defuzzification using the "centroid" method 

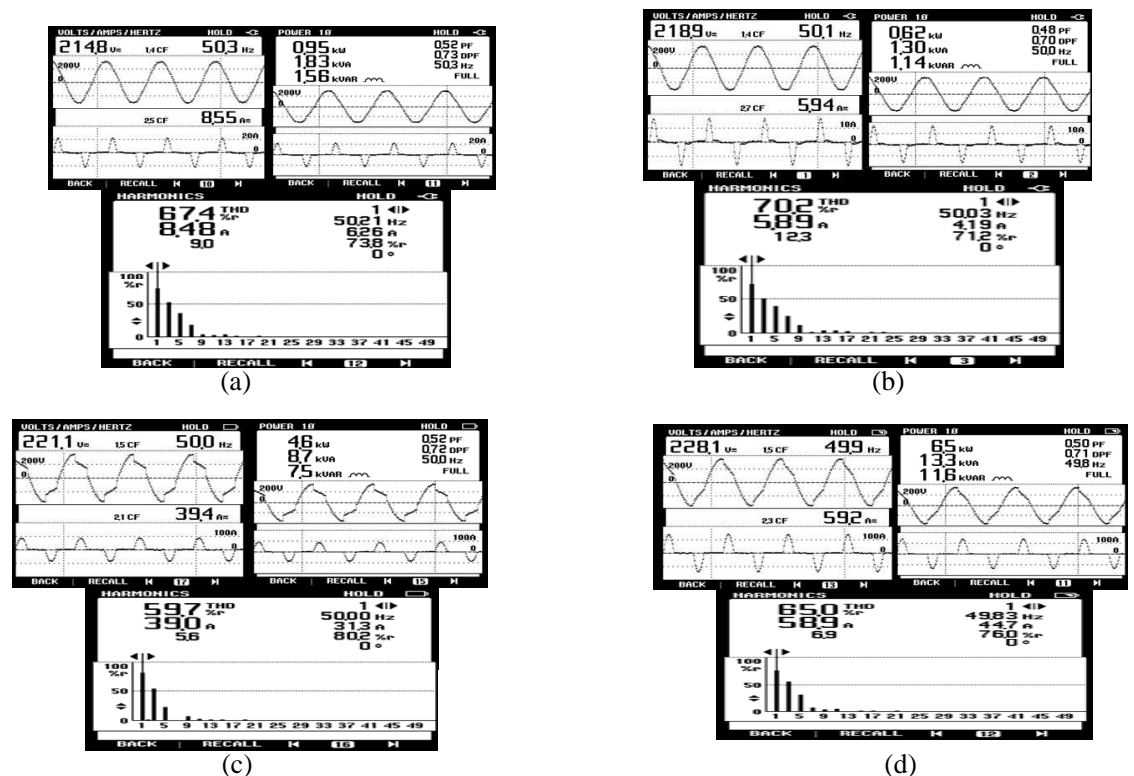

(d)

Fig. 1: Results of traditional UPS with (a), (c) 1 and 5kVA Linear load and (b), (d) 1 and 5 kVA Nonlinear load

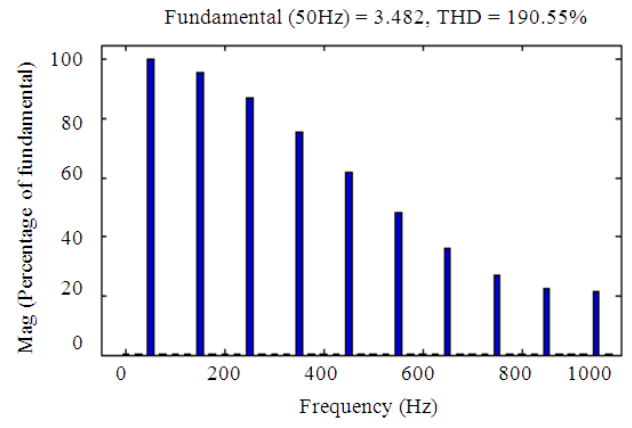

(a)

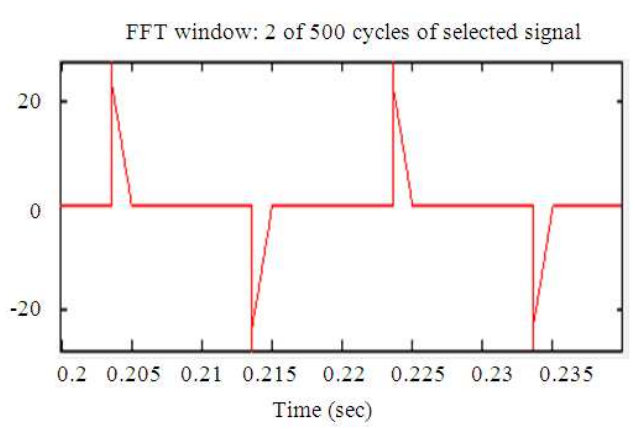

(b)

Fig. 2: FFT window of the source current and its profile for two selected cycles $\left(\mathrm{THD}_{\mathrm{i}}=190.55 \%\right)$

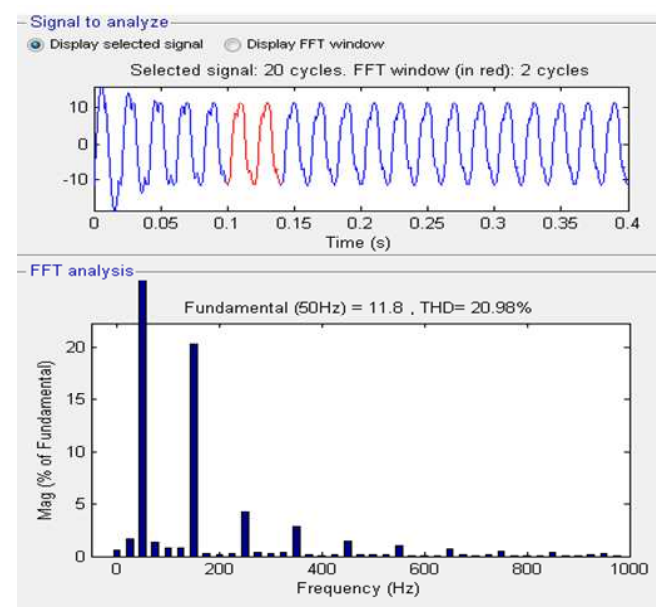

(a)

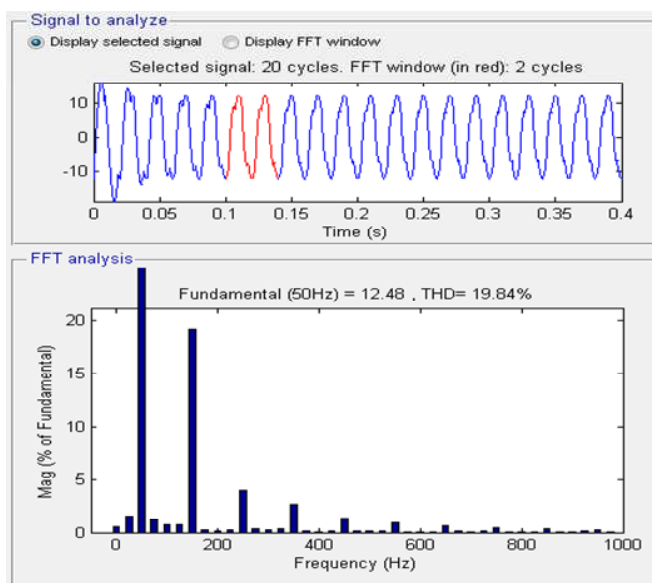

(b)

Fig. 3: Harmonic profile of Nonlinear Load (a) with ARF only (b) with ARF \& HPF 


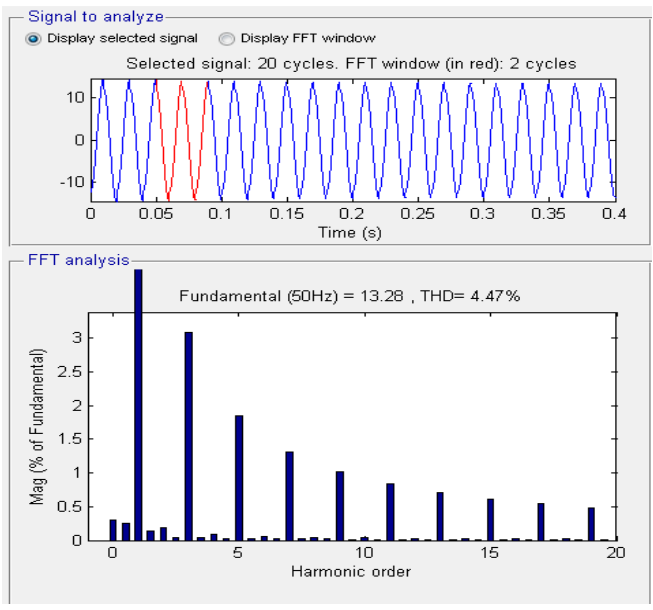

(a)

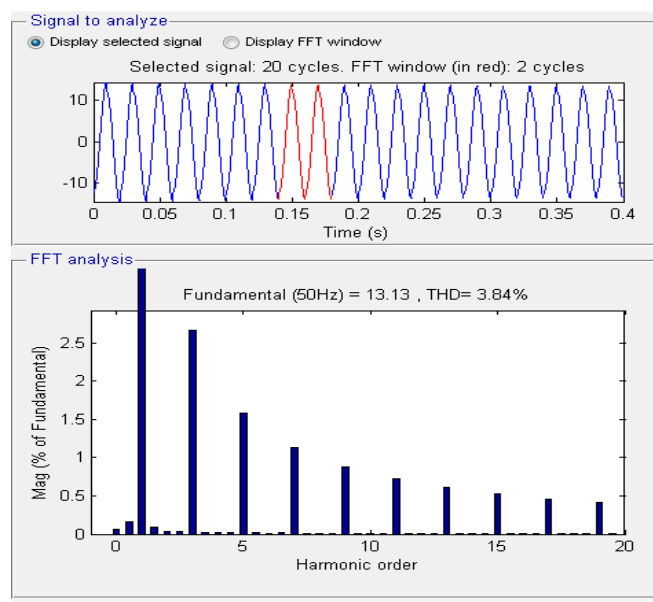

(b)

Fig. 4: Harmonic profile of Nonlinear Load (a) With ARF and FLC $\left(\mathrm{THD}_{\mathrm{i}}=4.47 \%\right)$ (b) With ARF and ANN $\left(\mathrm{THD}_{\mathrm{i}}=3.84 \%\right)$

Neuro trained controller with ARF: For improving the performance and obtaining better results the neural based current controller is implemented (Tey et al., 2005). The training of the network layer gives the required mitigation of harmonics (Sivanandan and Deepa, 2008). So for obtaining that harmonics reduction, back propagated neural network is used. This network is purely based on supervisory training.

The training uses gradient descent training method instead of steepest descent method of training. Since the adjustment for required output can be done continuously during the training process. The standard steepest descent is always used since the learning rate is held constant throughout training. But the total performance of this algorithm is very sensitive to the proper setting of the learning rate. It is not practical to determine the optimal setting for the learning rate before training and so the performance will better if the learning rate changes during training process. Thus it is done in adaptive gradient descent training method.

So for improving the performance, the learning rate is allowed to change during the training process. This adaptive learning rate attempts to keep the learning step size as large as possible while keeping learning stable.

\section{RESULTS}

The triple harmonic orders are reduced which shows the controllability of the controller. The harmonic profile shown above in the FFT window (Fig.
$3 \mathrm{a}$ and $\mathrm{b})$ gives the elimination of harmonics up to $\mathrm{THD}_{\mathrm{i}}=20.98 \%$ and $19.84 \%$. (Without and with HPF) compared to the uncompensated $\mathrm{THD}_{\mathrm{i}}$.

The fuzzy is implemented and rule base is utilized for effective harmonic alleviation. The rule base includes 25 rules and it defines the shape of the current to be for the supply. Thus fuzzy logic controller is employed and simulated. The results shown above in Fig. 4a shows the level of $\mathrm{THD}_{\mathrm{i}}$ by use of ARF with fuzzy controller $\left(\mathrm{THD}_{\mathrm{i}}=4.47 \%\right)$.

For improving the dynamic performance, adaptive learning NN (Chandr et al., 2000) is used with ARF. The harmonic reduction is done within the limits according to the standards $\left(\mathrm{THD}_{\mathrm{i}}=3.84 \%\right)$ shown in Fig. $4 \mathrm{~b}$.

\section{DISCUSSION}

The presence of harmonic distorts the wave form shape of the voltage and current, increases the current level and changes power factor supply, which in turn creates so many disturbances. Thus for safeguarding the systems from this kind of affected supply many harmonic alleviation techniques are used. Though many such techniques are proposed, they have their own demerits like (i) huge cost, (ii) extra additional components and (iii) controllers, independent of loads. Out of such techniques active filtering is now widely used for harmonic filtering but it differs from the proposed method of filtering by the way usage of extra shunt connected filter to reduce the harmonics. This study focuses on one such technique which has the 
advantage of (a) reduction of additional circuit (b) low cost (c) best harmonic reduction (both low \& higher order) without any shunt connected circuits and controllers. For having better performance artificial intelligent techniques are introduced with the proposed filter provide best solution for this harmonic pollution and it is also validated by using the simulation results with reduced $\mathrm{THD}_{\mathrm{i}}$ at the utility side.

\section{CONCLUSION}

The FFT profile clearly proves that all harmonic profiles especially for third harmonic profiles the regenerative converter with the current controllers gives effective mitigation of harmonics. The profile maintains the total harmonic distortion within the stringent limits of standards like IEEE 519 (ie., within 5\%). It is also shown that without any additional circuitry a regenerative converter is enough for better harmonic cancellation. The intelligent controllers in addition with that of Active regenerative filters help their best in giving harmonic pollution free green power from the utilities to all the loads.

\section{REFERENCES}

Benachaiba, C. and B. Ferdi, 2009. Power quality improvement using DVR. Am. J. Applied Sci., 6: 396-400. DOI: 10.3844/ajassp.2009.396.400

Chandr, A., B. Singh and B.N. Singh, 2000. An improved control algorithm of shunt active filter for voltage regulation, harmonic elimination, power-factor correction and balancing of nonlinear loads. IEEE Trans. Power Elect., 15: 495-507. DOI: $10.1109 / 63.844510$

Chen, T.C. and C.T. Pan, 1992. Modeling and design of a single phase ac to dc converter. IEEE Proc., 136: 465-470.

García, O. J.A. Cobos, R.P.P. Alou and J. Uceda, 2003. Single phase power factor correction: A survey. IEEE Trans. Power Elec., 18:749-755. DOI: 10.1109/TPEL.2003.810856

Ghosh, R. and G. Narayanan, 2005. A single-phase boost rectifier system for wide range of load variations. IEEE Trans. Power Elec., 22: 470-479. DOI: 10.1109/TPEL.2006.890011
IEEE, 1993. IEEE Recommended Practices and Requirements for Harmonic Control in Electric Power Systems. 1st Edn., Institute of Electrical and Electronics Engineers, New York, ISBN: 1559372397, pp: 100.

Kazerani, M., P.D. Ziogas and G. Joos, 1991. A novel active current wave shaping technique for solidstate input power factor conditioners. IEEE Trans. Indus. Elect., 38: 72-78. DOI: 10.1109/41.103488

Rabi, B.J. and R. Arumugam, 2005. Harmonics Study and Comparison of Z-source Inverter with Traditional Inverters. Am. J. Applied Sci., 2: 14181426. DOI: 10.3844 /ajassp.2005.1418.1426

Rodriguez, J.R., J.W. Dixon, J.R. Espinoza and J.P.P. Lezana, 2005. PWM regenerative rectifiers: State of the art. IEEE Trans. Indus. Elect., 52: 5-21. DOI: 10.1109/TIE.2004.841149

Salmon, J.C., 1993, Techniques for minimizing the input current distortion of current controlled single phase PWM rectifier. IEEE Trans. Power Elec., 8: 509-520. DOI: $10.1109 / 63.261022$

Sivanandan, D.S.N. and M.S.N. Deepa, 2008. Principles of Soft Computing, Wiley India (P) Ltd. ISBN: 13: 9788126510757

Tan, P.C., Z. Salam and A. Jusoh, 2005. A single-phase hybrid active power filter using extension p-q theorem for photovoltaic application. IEEE PEDS., pp: 1250-1254. DOI: 10.1109/PEDS.2005.1619879

Tey, L.H., P.L. So and Y.C. Chu, 2005. Improvement of power quality using adaptive shunt active filter. IEEE Trans. Power Delivery, 20:1558-1568. DOI: 10.1109/TPWRD.2004.838641

Valdez, A. G. Escobarand and R. Ortega, 2009. An adaptive controller for the shunt active filter considering a dynamic load and the line impedance. IEEE Trans. Cont. Syst. Technol., 17: 458-464. DOI: 10.1109/TCST.2008.2000987 